\title{
Relative Intensity Noise Transfer Reduction in Raman-Assisted BOTDA Systems
}

\author{
Xabier Angulo-Vinuesa, Denis Bacquet, Sonia Martin-Lopez, Pedro Corredera, Pascal Szriftgiser, \\ and Miguel Gonzalez-Herraez
}

\begin{abstract}
Raman-Assistance (RA) has been identified as a promising technique to extend the measurement range of Brillouin Optical Time-Domain Analysis (BOTDA)-based distributed sensors. Unfortunately, Raman amplification introduces a great amount of Relative Intensity Noise (RIN) to the detected low-frequency probe wave. This RIN transfer problem has been widely identified as a major limitation in RABOTDA. In Vector Brillouin Optical Time Domain Analysis (VBOTDA) the detected signal is transferred to a high-frequency carrier where the Raman RIN transfer turns out to be much less harmful. In addition, a VBOTDA can also provide information about the phase-shift induced by the local SBS gain curve, paving the way for dynamic measurements. In this work we demonstrate, for the first time to our knowledge, Ramanassistance in a VBOTDA obtaining gain and phase measurements. Our results show that a significant reduction of the RIN transfer effect in RA-VBOTDA compared to standard RA-BOTDA, making this type of scheme particularly interesting for long range and dynamic distributed sensing.
\end{abstract}

Index Terms - Brillouin scattering, distributed optic fiber sensor, distributed Raman amplification, phase detection.

\section{INTRODUCTION}

$\mathrm{B}$ Rillouin Optical Time Domain Analysis (BOTDA) [1] is now settled as one of the most employed distributed fiber optic sensor techniques for civil structure monitoring. A standard BOTDA can range up to approximately $50 \mathrm{~km}$. However, the request for long-range $(>50 \mathrm{~km})$ distributed fiber optic sensors for civil structure monitoring is progressively increasing due to the availability of new infrastructures crossing large unmanned areas. Thus, range increasing techniques have been investigated in order to achieve longer sensing distances with still one sensing head.

Manuscript received June 30, 2011; revised September 08, 2011; accepted September 08, 2011. Date of publication September 22, 2011; date of current version March 02, 2012.

X. Angulo-Vinuesa is with Focus S.L., Madrid 28004, Spain (e-mail: xabier.angulo@focustech.eu).

D. Bacquet and P. Szriftgiser are with the Laboratoire PhLAM, Université Lille 1, 59655 Villeneuve d’Asq, France (e-mail: pascal.szriftgiser@univlille1.fr; denis.baquet@phlam.univ-lille1.fr).

P. Corredera is with the Instituto de Óptica, Consejo Superior de Investigaciones Científicas, Madrid 28006, Spain (e-mail: pcorredera@io.cfmac.csic.es).

S. Martin-Lopez and M. Gonzalez-Herraez are with the Departamento de Electrónica, Universidad de Alcalá, Edificio Politécnico,Madrid 28871, Spain (e-mail: sonia.martin@depeca.uah.es; miguelg@depeca.uah.es).
Among these, one of the preferred techniques is RamanAssistance (RA), either using first-order pumping [2] or second-order pumping [3], [4]. These methods can help to achieve $100 \mathrm{~km}$ sensing range [2] and beyond [4] maintaining a considerably high resolution (2-3 meter) without any special kind of data treatment.

Unfortunately, distributed Raman amplification introduces Relative Intensity Noise (RIN) transfer to the detected signal, especially if Raman Fiber Lasers (RFL) are employed. This RIN transfer becomes especially harmful at lower frequencies $(<200 \mathrm{MHz})$ [2], which is the frequency region where the detection scheme of a standard BOTDA operates. Given its particular nature, it is usually difficult to average the transferred RIN noise, so special digital filtering techniques have been proposed for high-performance applications [5]. Overall, RIN transfer has been identified by many authors [6] as a major limitation in the performance of long-range Ramanassisted BOTDA sensors.

In this Letter, we show that Vector Brillouin Optical Time Domain Analysis (VBOTDA) [7] can provide a possible solution to avoid RIN transfer drawbacks when enhancing the range of distributed fiber optic sensors. In this technique, the probe wave is transferred to a high-frequency carrier region (> $500 \mathrm{MHz}$ ) where the RIN transfer from the Raman pump to the probe signal is strongly attenuated. The origin of this RIN transfer reduction lies in the large walk-off times between the Raman pumps and the BOTDA signals induced by chromatic dispersion (this walk-off amounts several periods of the carrier frequency in the VBOTDA). This causes any noise perturbation in the Raman pump to slip along several signal periods, amplifying the whole signal more homogeneously and reducing significantly its deleterious effect. Since the probe is phase modulated, through this technique it is also possible to construct a phase spectrogram from the phase time of flight. Phase measurements can be a convenient path to follow when trying to develop real time measurements [8] and, as has been recently reported by Urricelqui et al. [9], in order to avoid the appearance of non-local effects in terms of pulse depletion. Merging all these advantages could be considered a substantial breakthrough, since long-range, dynamic and robust measurements (> $50 \mathrm{~km}$ ) could be potentially achieved with this technique.

In this work we demonstrate, for the first time, Raman assistance in a VBOTDA. We provide experimental data showing that RIN transfer in a VBOTDA can be reduced by 
more than $10 \mathrm{~dB}$ over a conventional BOTDA in a bidirectional Raman amplification scheme. The results shown illustrate the potential of this technique for long-range measurements. Further noise reduction strategies could allow making long-range and dynamic measurements simultaneously.

\section{EXPERIMENTAL SETUP}

The developed experimental setup, which can be observed in Figure 1, is mainly based on introducing a first-order bidirectional Raman amplification module to the VBOTDA developed in [7].

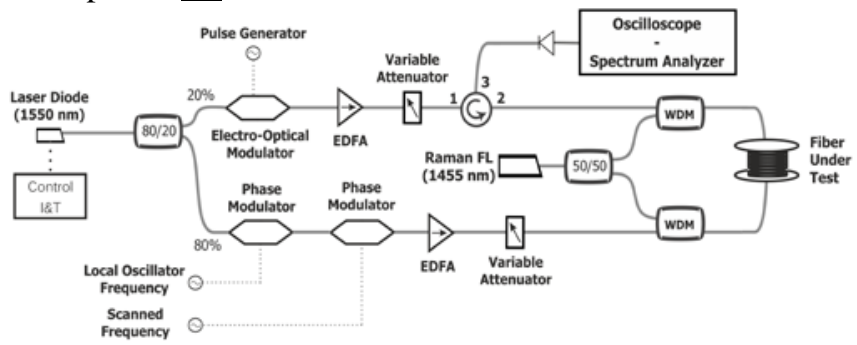

Fig. 1: Experimental setup of the VBOTDA system assisted by first-order Raman amplification. EDFA: Erbium Doped-Fiber Amplifier; WDM: Wavelength Division Multiplexer; FL: Fiber Laser.

The operation of a VBOTDA is similar to a standard BOTDA: a pulsed pump wave interacts locally with a frequency shifted continuous counter-propagating probe wave through Stimulated Brillouin Scattering (SBS) [10]. Generally both pump and probe signals are obtained from the same master source, which in this case is a low-noise $\sim 500 \mathrm{kHz}$ Distributed-FeedBack (DFB) laser diode emitting at $1550 \mathrm{~nm}$.

The pump wave is achieved by pulsing the continuous output of the DFB with 200 ns pulses (20 meter resolution) and afterwards amplified through an Erbium Doped Fiber Amplifier (EDFA) to obtain pulses with $\sim 3.5 \mathrm{~mW}$ peak power. The pulse width has been chosen to increase the contrast over the elevated Direct Current (DC) component. The low peak power is set so as to achieve results comparable, in terms of gain, to the ones obtained with a standard highresolution BOTDA ( $1 \%$ gain). However, we strongly believe that significant improvements could be obtained using higher extinction ratio pulsing elements such as SOAs [5].

In a standard BOTDA, the probe signal is achieved through intensity modulation, while in a VBOTDA the probe is created through two Phase Modulators (PM). These devices are going to be driven by one frequency each: a Local Oscillator Frequency ( $\left.F_{L O}\right)$, fixed at $750 \mathrm{MHz}$ in this case, far enough from the RIN transfer region and without significant Chromatic Dispersion impairments, and the so-called Scan Frequency $\left(F_{s}\right)$, which is swept around $10 \mathrm{GHz}$. In this way, the probe spectrum is therefore going to be formed by the following frequency couples: $\pm \mathrm{F}_{\mathrm{S}}, \pm\left(\mathrm{F}_{\mathrm{S}}-\mathrm{F}_{\mathrm{LO}}\right)$ and $\pm\left(\mathrm{F}_{\mathrm{S}}+\mathrm{F}_{\mathrm{LO}}\right) \underline{\text { as }}$ represented in Figure 2. This last frequency is roughly equal to the Brillouin shift of the fiber $\left(\mathrm{F}_{\mathrm{BGS}}\right)$, and the scanning of $\mathrm{F}_{\mathrm{S}}$ will allow to scan this frequency over the Brillouin Gain (BG) of the fiber under test. In this scheme, a significant component at the pump frequency will also appear, which introduces significant noise as we will see later. Different schemes could be used in order to avoid this issue, as will be discussed later. Under no Brillouin Gain, the detected beat note between $F_{S}$ and $\mathrm{F}_{\mathrm{S}}+\mathrm{F}_{\mathrm{LO}}$ is completely compensated by the beat note between $\mathrm{F}_{\mathrm{S}}$ and $\mathrm{F}_{\mathrm{S}}-\mathrm{F}_{\mathrm{LO}}$ since they have opposite phases. Yet, if $F_{S}$ is arranged so that $F_{S}+F_{L O}$ is located around the Brillouin Gain Spectrum (BGS) of the fiber, then the $-\left(\mathrm{F}_{\mathrm{S}}+\mathrm{F}_{\mathrm{LO}}\right)$ sideband will be amplified and $+\left(\mathrm{F}_{\mathrm{S}}+\mathrm{F}_{\mathrm{LO}}\right)$ attenuated. The phase modulation imbalance will then produce an intensity beat note at $F_{L O}$. The detection of the amplitude of the intensity modulated $\mathrm{F}_{\text {LO }}$ frequency as a function of time through a high-bandwidth photo-detector will provide both phase and amplitude information of the BGS [9]. In this case, the probe signal power is also amplified to obtain a power level $\sim 40 \mu \mathrm{W}$.

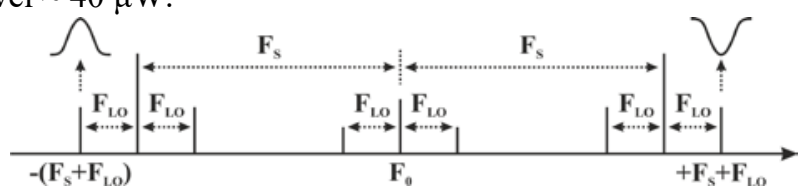

Fig. 2: Double-frequency phase modulation principle. FLO: Local Oscillator Frequency; FS: Scanned Frequency; \pm (FS+FLO): Amplification or Attenuation Sidebands.

The selected power levels ensure a proper trade-off between the detected signal level and the prevention of undesired nonlinear effects as well as pump depletion. Together with the pump and probe waves, a Fiber Laser (FL) emitting at 1455 $\mathrm{nm}$, which amplifies all the signals within the fiber, is introduced in the scheme to provide the distributed Raman amplification. This laser presents a relatively high RIN ( -110 $\mathrm{dBc} / \mathrm{Hz}$ ) which might be transferred to the probe wave.

As it has been said before, the employed setup had a considerable DC power level leakage due to the impossibility to minimize the carrier frequencies of the PM properly and the several modulated signals present within the fiber. This last issue could be avoided by using a unique side-band PM scheme [10]. These setbacks degraded the performance of the setup forcing us to employ low power pump signals with considerably long pulse widths (200 ns), together with Raman amplification for a relatively low sensing range for the present BOTDA performance technology. Although this could be considered as a drawback, it does not affect the main conclusions of this work as it does not affect the demonstration of the reduction of the RIN transfer effects caused by the Raman assistance.

The complete setup can also be considered as an opening gate to real-time measurements in long range BOTDA systems thanks to the steep linear phase response of the interaction around the center frequency [8].

\section{RESULTS}

The verification of the RIN transfer reduction was developed by comparing the whole frequency spectrum of the non-modulated probe signal under Raman amplification with the normalized spectral power density of the probes noise without Raman assistance. As a simple test, the bi-directional Raman amplification was set at $550 \mathrm{~mW}$, which means 275 
$\mathrm{mW}$ of Raman amplification on the probe side. Figure 3 shows the $\mathrm{FFT}^{2}$ (squared Fast Fourier Transform) representation of the detected probe wave RIN noise as a function of frequency. As it can be clearly seen, at low frequencies $(<200 \mathrm{MHz})$, where a standard BOTDA operates, the probe RIN level increases around $30 \mathrm{~dB}$ when the Raman pump is turned on (blue trace). By setting the probe wave at $750 \mathrm{MHz}$ it can be seen that the noise increase given by Raman pumping is reduced to $20 \mathrm{~dB}$, which implies a $10 \mathrm{~dB}$ reduction in terms of transferred RIN noise. As the frequency increases the noise is reduced even more (15 $\mathrm{dB}$ reduction at $1 \mathrm{GHz}$ ), however at higher frequencies the chromatic dispersion effect introduces a non-negligible PM-AM conversion in the probe signal [10]. In case of ambient variations, the optimum probe modulation frequency should not vary since no extreme RIN or chromatic dispersion drifts are expectable. This operating regime would also restrict the VBOTDA dynamic range. Therefore, it is necessary to find a proper trade-off between the RIN reduction achieved and the dynamic range reduction caused by chromatic dispersion. For the experimental settings, we set $\mathrm{F}_{\mathrm{LO}}$ $=750 \mathrm{MHz}$.

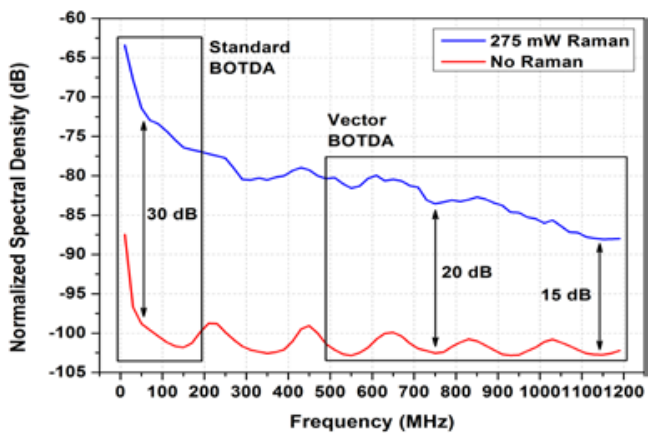

Fig. 3: Comparison of the spectral power density of the probe intensity noise with and without Raman assistance when the amplification is set at $550 \mathrm{~mW}$ in a bi-directional configuration ( $275 \mathrm{~mW}$ for the probe signal).

In the settings of Figure 2, the overall Raman gain introduced in the probe is roughly $17 \mathrm{~dB}$. In conventional BOTDA conditions, the RIN increase (measured with the $\mathrm{FFT}^{2}$ ) is $30 \mathrm{~dB}$ (which implies roughly $15 \mathrm{~dB}$ noise amplitude growth) and therefore the Raman gain only introduces a $2 \mathrm{~dB}$ improvement in overall signal to noise ratio. In the VBOTDA region, the noise amplitude growth goes down to $10 \mathrm{~dB}$, implying a $7 \mathrm{~dB}$ growth in optical SNR. This should lead to a clear improvement in performance.

Once the RIN transfer reduction was proved and selected $750 \mathrm{MHz}$ as the proper modulation for our purposes, the performance of the Raman-assisted VBOTDA as a sensor was tested. $84.5 \mathrm{~km}$ of fiber were monitored, formed by three spools spanning $40 \mathrm{~km}, 42.5 \mathrm{~km}$ and $2 \mathrm{~km}$ respectively, of which the first two had a maximum Brillouin Frequency Shift (BFS) of $10.86 \mathrm{GHz}$ at room temperature $\left(\sim 20^{\circ} \mathrm{C}\right)$ and the last one $(2 \mathrm{~km})$ had a BFS of $10.88 \mathrm{GHz}(20 \mathrm{MHz}$ difference from the first two spools). The sensor performance was checked by introducing the last $2 \mathrm{~km}$ of the total length in a temperature controlled oven at $60{ }^{\circ} \mathrm{C}$ and keeping the remaining $82.5 \mathrm{~km}$ of fiber at $20{ }^{\circ} \mathrm{C}$ constant controlled temperature. The $40{ }^{\circ} \mathrm{C}$ temperature difference is translated as approximately $40 \mathrm{MHz}$ frequency shift $\left(0.001 \mathrm{GHz} /{ }^{\circ} \mathrm{C}\right)$ [17]; thus, we expect to observe a 20 meter transition of $60 \mathrm{MHz}$ $(20 \mathrm{MHz}+40 \mathrm{MHz})$ at the $82.5 \mathrm{~km}$ position from $10.86 \mathrm{GHz}$ until $10.92 \mathrm{GHz}$.

In Figure 4, the whole $84.5 \mathrm{~km}$ demodulated amplitude traces $(82.5 \mathrm{~km}+2 \mathrm{~km})$ are shown, at the frequencies of 10.86 $\mathrm{GHz}$ and $10.92 \mathrm{GHz}$ (corresponding to the BGS at $20^{\circ} \mathrm{C}$ and $60{ }^{\circ} \mathrm{C}$ ). The acquisition is done with 500 averages. A clear gain shift in the end of the fiber is noticeable, and the contrast between the heated and non-heated sections is very good.

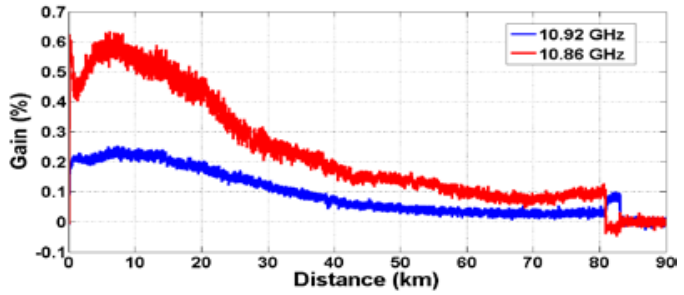

Fig.4Fig. 1: Whole trace measurement (spectrum analyzer with zero-span mode) for $10.86 \mathrm{GHz}$ (red trace) and $10.92 \mathrm{GHz}$ (blue trace). At the expected location $(82.5 \mathrm{~km})$ a clear gain transition is observed, and the maximum gain at the fiber end is shifted as expected.

Based on previous results obtained with standard BOTDA systems over similar sensing distances (between 75 and 100 $\mathrm{km})$ [2], [5], and similar gain values $(<1 \%)$, it can be concluded that the RIN transfer is considerably reduced since the results have a neat determination with a relatively low number of averages. The fact that we could not increase the pump power significantly implied a significant loss in terms of achievable resolution. Similar gain values $(<1 \%)$ can be obtained with a high extinction ratio pulsing method ( $>40$ $\mathrm{dB}$ ), a tenfold increase in peak power and a tenfold reduction in pulse width (see e.g. [5]). However, the measurement of such low gain values would require thousands of averages in a conventional Raman-assisted BOTDA [2],[5].

A detailed gain and phase sweep of the transition between the heated and non-heated sections is seen in Figure 5 a) and b) respectively. A complete switch of the gain position can be seen around the position of $82.5 \mathrm{~km}$. As can be seen, the complete gain switch is achieved in 20 meters, confirming the aforementioned resolution values, as it is detailed in Figure 6 . This measurement was developed in a 200 meter span through an oscilloscope. The measurement was developed trying to obtain a neat trace with the minimum number of averages possible, so as to illustrate the good RIN transfer capabilities of the setup. In this case 512 averages were employed, which is less than 10 times lower than the number of averages employed with standard BOTDA sensors for similar sensing distances and gain values [2],[5]. It is noticeable that the phase measurement frequencies shows maxima that are slightly lower than the ones obtained from the gain trace. This arises since the phase shift at the maximum gain frequency experiences a steep switch from maximum to minimum phase around the maximum frequency, the maximum phase shift being approximately $20 \mathrm{MHz}$ lower than the peak gain. As it is visible, the frequency difference perfectly matches the expected $60 \mathrm{MHz}$. 
a)

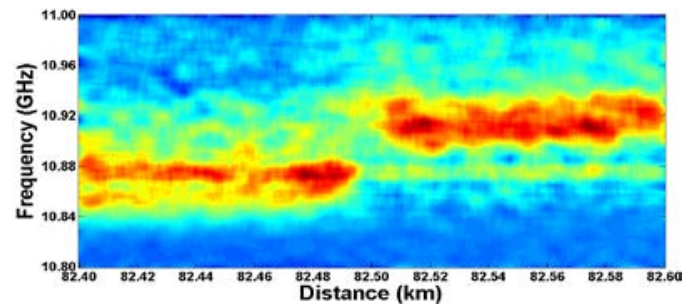

b)

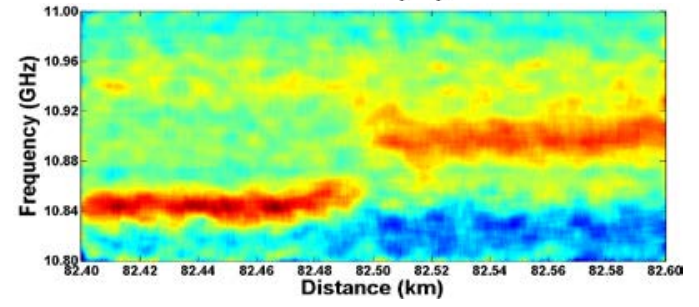

Fig. 5: 200 meter zoom (oscilloscope) at the heated fiber transition zone for a) gain and b) phase measurements. A 20 meter transition can be seen at the expected location from $10.85 \mathrm{GHz}$ until $10.91 \mathrm{GHz}$ (from $10.84 \mathrm{GHz}$ until $10.90 \mathrm{GHz}$ for the phase), which corresponds to the expected $60 \mathrm{MHz}$ transition $(20 \mathrm{MHz}+40 \mathrm{MHz})$.

a)

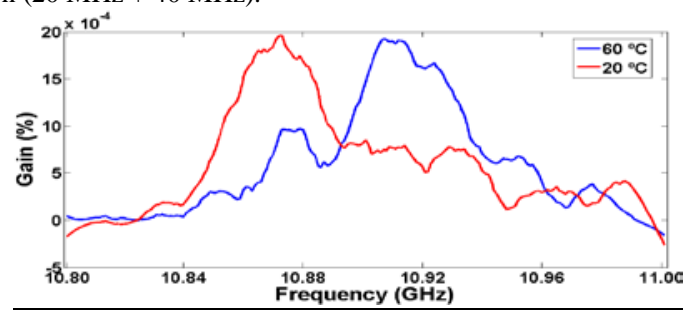

b)

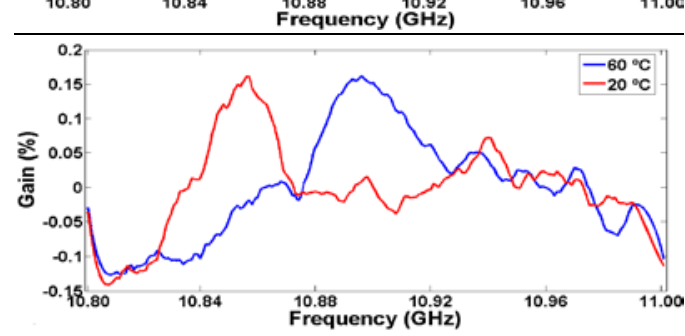

Fig. 6: Experimental representations of the achieved gain traces for the developed whole frequency span $(10.80 \mathrm{GHz}-11 \mathrm{GHz})$ at $20^{\circ} \mathrm{C}$ and $60^{\circ} \mathrm{C}$ (red and blue trace respectively) for a) gain and b) phase.

\section{CONCLUSIONS}

In this work we have demonstrated for the first time firstorder Raman assistance in a VBOTDA. Compared to a standard BOTDA, the VBOTDA uses a high-frequency (> 500 $\mathrm{MHz}$ ) modulated probe wave. In terms of Raman assistance, this allows reducing considerably the RIN transfer effect to the detected amplified probe wave, which is one of the major limitations in Raman-assisted Brillouin distributed sensors. The measured noise reduction can be as large as $10 \mathrm{~dB}$. Further reduction could be achieved at higher frequencies; however a trade-off remains to be found because chromatic dispersion causes some undesired PM-AM conversion in the probe wave at higher frequencies and reduces the dynamic range of the measurement accordingly [11]. In addition to the impairments due to chromatic dispersion, it is also remarkable the deleterious effect on the measurement noise caused by Four-Wave Mixing (FWM) among the different probe wave components. It should be considered that the spectral components in the probe wave are polarization-aligned and close in frequency: in these conditions FWM is maximized, as the phase mismatch vanishes [10]. Nonetheless, the performance of the setup as a sensor was neatly verified with furthermore very low averaging ( 500) compared to a standard BOTDA with similar gain and range values $(<1 \%$ peak gain, $85 \mathrm{~km}$ ), proving the potential of this technique in long-range and real-time distributed measurements. We believe that the combination of the proposed technique together with Semiconductor Lasers (SL), which has been identified by Soto et al. as a RIN transfer lowering technique [6], could considerably increase the performance of low noise long range BOTDA systems. Further work should concentrate in solving the issues associated to chromatic dispersion and FWM. A possible strategy to follow could be the use of a single sideband modulation in the probe wave as proposed by Urricelqui et al. [9].

\section{ACKNOWLEDGMENTS}

We acknowledge funding from the European Research Council through Starting Grant U-FINE (Grant no. 307441), the Spanish "Plan Nacional de $\mathrm{I}+\mathrm{D}+\mathrm{i}$ " through projects TEC2012-37958-C02-01 and TEC2012-37958-C02-02, the regional program FACTOTEM2 (S2009/ESP-1781) funded by the Comunidad de Madrid, the INTERREG SUDOE program ECOAL-MGT and the Nord-Pas de Calais Regional Council and FEDER through the Contrat de Projets Etat Région “Campus Intelligente Ambiante” (CPER-CIA) 2007-2013.

\section{REFERENCES}

[1] T. Horiguchi, S. Shimizu, K. Kurashima, M. Tateda, and Y. Koyamada, "Development of a distributed sensing technique using Brillouin scattering,” IEEE J. Lightwave Technol., vol. 13, no. 7, pp. 1296-1302, 1995.

[2] X. Angulo-Vinuesa, S. Martin-Lopez, J. Nuño, P. Corredera, J. D. AniaCastañon, L. Thévenaz, and M. Gonzalez-Herraez, "Raman-assisted Brillouin distributed temperature sensor over $100 \mathrm{~km}$ featuring $2 \mathrm{~m}$ resolution and $1.2^{\circ} \mathrm{C}$ uncertainty," IEEE J. Lightwave Technol., vol. 30, no. 8, pp. 1060-1065, 2012.

[3] S. Martin-Lopez, M. Alcon-Camas, F. Rodriguez-Barrios, P. Corredera, J. D. Ania-Castañon, L. Thévenaz, and M. Gonzalez-Herraez, "Brillouin optical time-domain analysis assisted by second-order Raman amplification," Opt. Express, vol. 18, no. 18, pp. 18769-18778, 2010.

[4] X. Angulo-Vinuesa, M. A. Soto, S. Martin-Lopez, S. Chin, J. D. AniaCastañon, P. Corredera, E. Rochat, M. Gonzalez-Herraez, and L. Thévenaz, "Brillouin optical time-domain analysis over a $240 \mathrm{~km}$-long fiber loop with no repeater,” Proc. SPIE, vol. 8421, no. 8421C9, 2012.

[5] X. Angulo-Vinuesa, S. Martin-Lopez, P. Corredera, and M. GonzalezHerraez, "Raman-assisted Brillouin optical time-domain analysis with sub-meter resolution over $100 \mathrm{~km}$," Opt. Express, vol. 20, no. 11, pp. 12147-12154, 2012.

[6] M. A. Soto, M. Taki, G. Bolognini, and F. Di Pasquale, "Simplex-coded BOTDA sensor $120 \mathrm{~km}$ SMF with $1 \mathrm{~m}$ spatial resolution assisted by optimized bidirectional Raman amplification," IEEE Photon. Technol. Lett., vol. 24, no. 20, pp. 1823-1826, 2012.

[7] M. Dossou, D. Bacquet, and P. Szriftgiser, "Vector Brillouin optical time-domain analyzer for high-order acoustic modes," Opt. Lett., vol. 35, no. 22, pp. 3850-3852, 2010.

[8] J. Urricelqui, A. Zornoza, M. Sagues, and A. Loayssa, "Dynamic BOTDA measurements based on Brillouin phase-shift and RF demodulation," Opt. Express, vol. 20, no. 24, pp. 26942-26949, 2012.

[9] J. Urricelqui, M. Sagues, and A. Loayssa, "BOTDA measurements tolerant to non-local effects by using a phase-modulated probe wave and RF demodulation,” Opt. Express, vol. 21, no. 4, pp. 17186-17194, 2013.

[10] G. P. Agrawal, "Nonlinear Optics", 4th ed. CA: Academic 2007.

[11] W. K. Marshall, B. Crosignani, and A. Yariv "Laser phase noise to intensity noise conversion by lowest-order group-velocity dispersion in optical fiber: exact theory," Opt. Lett., vol. 25, no. 3, pp. 165-167, 2000. 